Di-Luoffo et al

\title{
The biomechanical context influences the output signaling, independently of PIK3CA mutations in breast cancer cells.
}

\author{
Di-Luoffo M.1,2, Delarue M.2, Guillermet-Guibert J. 1,*
}

$1 \quad$ Affiliation 1; INSERM U1037

2 Affiliation 2; LAAS-CNRS

* $\quad$ Correspondance: julie.guillermet@inserm.fr; Tel.: +33582741652

Abstract: Mechanical stresses including tensile and compressive stresses are ubiquitous in nature, and are now well-recognized as being inherent to the development of most cancers. They are integrated by mechanotransduction in cells. Tensile stress is largely associated with YAP/TAZ pathway activation. However, less is known about signaling induced by compressive stress, the latter arising from extracellular matrix remodeling and local tumor growth. In the present study, we used transcriptomic data obtained after unidirectional compression of wildtype and mutant PIK3CA breast cancer cells from Kim et al., 2019. We analyzed in an unbiased manner signatures of cell signaling activation including phosphoinositide 3-kinases (PI3Ks) activity pathway in response to compressive stress. Because we found that PI3K activation occurred upon compression, we studied PI3K isoform-specific pathways using known transcriptional targets of PI3K $\alpha$ inhibitor (BYL719) or of PI3K $\beta$ inhibitor (AZD8186). Our study provides transcriptomic evidences for the role of PI3K pathway in compression-induced mechanotransduction, through the roles of isoform-specific class I PI3Ks and independently of PIK3CA alterations. In a compressive environment, the canonical pathways (YAP/TAZ and Piezo) was not increased, while other targetable mechanisms, such as PI3K signal or autophagy, may provide a proliferative advantage and increased cell resistance to chemotherapies.

Keywords: PI3K-AKT, PI3K $\alpha$, PI3K $\beta$, compressive stress, mechanotransduction, autophagy. 
Di-Luoffo et al

\section{Introduction}

In tissues, all cells are subjected to mechanical forces, exerting a stress on the cell surface, cytoplasm or nucleus. Physically, this stress is measured with internal tissue/cell resistance to deformation described in $\left(\mathrm{N} / \mathrm{m}^{2}\right)$ or Pascals (Pa). Cells encounter 3 types of mechanical stresses: shear, tensile and compressive stress [1]. These mechanical interactions can emerge from cellcell or cell-substrate interaction [2]. Shear stress occurs when mechanical forces are applied parallel to a surface such as the fluid shear stress arising from blood flow which physically stresses blood vessel walls. In contrast, tensile and compressive mechanical stresses are inherent of an organ. In physiological condition, cells are subjected to minimal compression stresses. In solid tumor development, compression induced by cell contacts and interactions is increased, as tumor grow in a limited space. The impact of compressive stress on tumor progression and migration are dependent on the magnitude, duration and direction of applied forces, and is associated to extracellular tissue components [1]. In vitro, in most cases, 3D models of solid cancer cells under compressive stress show a cell proliferation decrease. However, compression can increase cancer cell invasive capabilities as well as their resistance to chemotherapeutic treatments due to a continued cell survival and proliferation [3-5]. Currently, there is no mean to predict what will be the cellular output of compression in cancer cells (decreased or increased proliferation and migration).

Once sensed by cells, a mechanical stress induces a mechanotransduction response, coupled to alteration of gene expression, which was largely associated with Hippo pathway activation under tensile stress [6]. In tumors, Hippo pathway containing transcriptional regulators YAP/TAZ can reprogram cancer cells into cancer stem cells and incite tumor initiation, progression and metastasis [6]. Further, the Hippo pathway crosstalks with morphogenetic signals, such as Wnt growth factors, and is also regulated by Rho and G protein-coupled receptor (GPCR), cAMP and PKA pathways [7]. These pathways were very well studied individually. However, in the last decade, research has shown the interconnections of signaling pathways, and key pathways involved in mechanotransduction were noticed [8]. In this context, the importance of phosphoinositide 3-kinases (PI3Ks) activity in mechanotransduction in cancers was misestimated. Recent evidence showed their implication as a pivotal role in mechanotransduction [8].

PI3K proteins can be divided into three classes (I-III) based on their primary structure, regulation, and in vitro lipid substrate specificity [9]. In the literature, the term PI3K refers usually to class I PI3K. This class, composed of four enzymes $(\alpha, \beta, \delta, \gamma)$, with nonredundant functions [9, 10] and nonredundant roles in cancer [11], generates phosphatidylinositol 3,4,5trisphosphate (PtdIns-3,4,5-P3 or PIP3) from phosphatidylinositol 4,5-bisphosphate (PtdIns4,5-P2 or PIP2) [9]. This reaction is reversed by the phosphatase activity of Phosphatase and TENsin homolog (PTEN). In pancreatic cancer cells, compression-induced PI3K activation promotes migratory phenotype involving an autocrine autostimulation loop [4, 12]. Class I PI3Ks are known to be upstream activators of YAP/TAZ transcriptional pathway under tensile stress, positioning class I PI3Ks proteins as upstream regulators of an essential mechanotransduction signaling [13]. Furthermore, in breast cancer cells, in vivo overexpression of PI3K $\beta$ sensitizes untransformed cells to YAP/TAZ-induced oncogenicity [13]. Furthermore, PI3K pathway is one of the most common aberrantly activated pathway in PIK3CA mutated breast cancer cells which provided the rationale for development of inhibitors targeting PI3K-AKT pathway (reviewed in [14]).

While the role of tensile stress is now largely recognized as affecting tumor cell fate [15], the role of compression stress is emerging. In particular, the global cell signaling modification in response to compression has been poorly characterized. Hence, we decided to use the 
Di-Luoffo et al

transcriptomic data obtained after unidirectional compression of breast cancer cells available to date (Kim et al., 2019 [16] to analyze in an unbiaised manner the signatures of cell signaling activation including PI3K pathway. Because, we found that PI3K activation occurred upon compression, we studied PI3K isoform-specific pathways using known transcriptional targets of PI3 K $\alpha$ inhibitor (BYL719) (Bosch et al., 2015 [17]) or of PI3K $\beta$ inhibitor (AZD8186) (Lynch et al., 2017 [18]). Here, we confirmed that PI3K pathway merits a greater attention as mechanical sensor of compression and that breast cancer cells are excellent models to study overexpression and activation of PI3K signaling [8].

\section{Results}

\subsection{PI3K-AKT is a key mechanosensitive pathway under compression in breast cancer cells}

We first aimed at analyzing how compressive stress altered signature of cell signaling pathways. For this, we searched for datasets obtained upon compression of cancer cells in Gene Expression Omnibus database (GEO), where public sequencing data from published studies are available (Figure. 1A). In GEO database, limited transcriptomic data obtained after cell compression are currently available. Kim et al. [16] performed breast cancer cell and cancer associated fibroblast (CAF) compressions using alginate disks applied on top of 2D cell layers. In this study, Kim et al. focused on and analyzed the action of compression on stromal cells. They observed that mechanical stress in these cells promoted a specific metabolic gene signature increasing glycolysis and lactate production [19]. Here, we investigated compression-induced mechanotransduction in cancer cells. Interestingly, in order to elucidate the gene expression evolution of breast cancer cells under compression, Kim et al. based their analysis on two cancer cell lines with two different genetic alteration profiles. The MDA-MB-231 cell line with a PI3K wild-type catalytic domain (PIK3CAWT) was compared to MCF-7 containing a constitutive PI3K activation (PIK3CAE545K). Both breast cancer cell lines were compressed from 0 to $8 \mathrm{kPa}$, and gene expression profile was analyzed. We first searched for gene signature enrichment analysis using canonical signatures from Gene Set Enrichment Analysis (GSEA) (Figure. 1A). Gene signatures in different pathways were normalized into transcripts per million and compared to housekeeping gene expression such as ACTINB, LAMINA1 and LAMIN gene class. The expressions of the latter were not affected by increasing compression (Figure. S1A). In wild-type PI3 KCA MDA-MB-231 cells submitted to high compression (8kPa), the NOTCH, MAPK and PI3KAKT were found as most enriched pathways compared to no compressed condition ( $0 \mathrm{kPa})$ (Figure. 1B), showing that few genes belonging to these signature had significant increased expression. This pathway enrichment was not found in MCF-7 cells presenting a constitutively activated PI3K $\alpha$. In addition, under increasing compression [0], [0.3-0.8], [1.5-4.0], [7.0-8.0] kPa; NOTCH, MAPK, and PI3K-AKT signatures mean gene expression (from GSEA canonical signatures) were significantly overexpressed only for MAPK and PI3K-AKT pathways in wildtype PIK3CA MDA-MB-231 cells compared to constitutively active PI3K MCF-7 cells (Figure. 1C), suggesting a global overexpression of all genes belonging to these pathways. Contrariwise, MCF7 cells showed a significant decrease for NOTCH and MAPK pathway gene expressions under increasing compressive stress (Figure. 1C). However and surprisingly, YAP/TAZ pathway was not significantly affected in MCF-7 and MDA-MB-231 cells (Figure. S1B). Taken together, these data showed a sensitivity (increasing gene expression) to growing compression (from 0 to 8 $\mathrm{kPa}$ ) in wild-type PI3KCA cells compared to overactivated PIK3CA cells. 
Di-Luoffo et al

This sensitivity in signaling pathway expression adds an additional link to demonstrate general PI3K involvement in mechanotransduction.

\section{$2.2 \mathrm{PI} 3 \mathrm{~K} \alpha / \beta$ are sensitive to compression in breast cancer cells}

To investigate the specific role of PI3K isoforms, we analyzed data from two studies which used selective inhibitors of PI3K isoforms: Bosch et al., 2015 [17] and Lynch et al., 2017 [18]. The authors used BYL719 and AZD8186 compounds to selectively inhibit PI3K $\alpha$ or PI3K $\beta$ in mutant PIK3CA MCF-7 and mutant PTEN HCC70 breast cancer cells respectively (Figure. 1A). We know that mutant PTEN leads to increased PI3K activity. Gene expression data from PI3 K $\alpha$ inhibition in MCF-7 and PI3K $\beta$ inhibition in HCC70 cells were used to define PI3K $\alpha$ and PI3K $\beta$ signatures. Selective transcriptional targets were crossed-compared with compression-specific transcriptional targets identified from Kim et al. [16] (Figure. 1A).

We analyzed data after PI3K $\alpha$ inhibition and we observed 1279 genes with a significantly altered expression in MCF-7. After PI3K $\beta$ inhibition in HCC70 cells, gene expression of 933 genes was also significantly affected. Gene expression of 1052 targets was affected in compressive conditions ([0.3-0.8], [1.5-4.0] and [7.0-8.0] kPa compared to [0] $\mathrm{kPa}$ ) in MCF-7 cells. We compared these signatures to the list of the 102 genes found in canonical "PI3K-AKT GSEA signaling pathway in cancer" signature (Figure. 2). PI3K $\alpha$ and PI3K $\beta$ signatures overlapped with the regulation of 139 genes (listed in Figure. 2, top table). Compressive stress gene signature mostly overlapped either with PI3K $\alpha$ or to PI3K $\beta$ signatures, on non common 32 and 31 genes respectively (listed in Figure. 2, left and right tables respectively), suggesting a differential effect on isoform activation in response to compression. We next found that both PI3K $\alpha$ and PI3K $\beta$ gene signatures (after PI3K $\alpha$ or PI3K $\beta$ inhibitions) were significantly overexpressed under increasing compression in wild-type PI3K MDA-MB-231 cells, but significantly decreased in PI3K oncogenically activated MCF-7 cells (Figure. 3 ).

These analyses showed that compression and PI3K signaling share common transcriptional targets, and that PI3 K $\alpha$ and PI3K $\beta$ gene signatures are altered upon compression, regardless the mutational status of PIK3CA.

\subsection{Compression influences autophagy gene expression in breast cancer cells}

PI3K $\alpha$ and PI3K $\beta$ are known as regulators of autophagy process [20, 21]. When comparing PI3K $\alpha$ and PI3K $\beta$ signatures, PI3K-AKT GSEA canonical pathway and compressive stress selective gene expression, only GABA type A receptor associated protein like 1 (GABARAPL1) gene was significantly affected and overlap in these four signatures (Figure. 2). Briefly, this gene encodes for a structural protein of autophagosome and autophagolysosome involved in autophagy process (reviewed in [22]). GABARAPL1 gene expression was significantly upregulated after PI3K $\alpha$ (x1.90; p-value: 1.83.10-2) and PI3K $\beta$ (x1.42; p-value: 6.15.10-3) inhibitions. Under increasing compression from [0] $\mathrm{kPa}$ up to [7.0-8.0] $\mathrm{kPa}$, GABARAPL1 gene expression was overexpressed in wild-type PI3K MDA-MB-231 cells, but not in constitutively PI3K-activated MCF-7 cells (Figure. 3B), suggesting a differential coupling of PI3K signal to GABARAPL1 expression under compression. Moreover, under increasing compression, the canonical autophagy GSEA pathway gene expression significantly increased in wild-type PI3K MDA-MB-231 cells, and significantly decreased in constitutive PI3K activated MCF-7 cells (Figure. 3C).

Therefore, autophagy GSEA pathway and GABARAPL1 gene expressions were well correlated and overexpressed in wild-type PIK3CA MDA-MB-231 cells upon compression. 
Di-Luoffo et al

\section{Figures, Tables and Schemes}

Figure 1. PI3K-AKT: a pathway involved in mechanotransduction in breast cancer cells. A. On the left side, the canonical method consisting in Gene Set Enrichment Analysis (GSEA) using canonical signatures. Available transcriptomic data from compressed breast cancer cells were used (Gene Expression Omnibus data (GEO) Kim et al., 2019. Breast cancer cells (MDA-MB-231: PIK3CAwt and MCF-7: (PIK3CAE545K mutated) were compressed from 0 to $8 \mathrm{kPa}$ and then a differential expression analysis was performed between 0 ; [0.3 to $0.8 \mathrm{kPa}$ ]; [1.5 to $4.0 \mathrm{kPa}$ ]; [7.0 to $8.0 \mathrm{kPa}$ ] respectively. In parallel, differential expression analysis were performed from breast cancer cells treated with two PI3K inhibitors (PI3K $\alpha$ (BYL719) and $\beta$ (AZD8186)) (Bosch et al., 2015 and Lynch et al., 2017). The gene expression profiles after PI3K $\alpha$ and $\beta$ inhibitions (PI3K $\alpha$ and $\beta$ signatures) were compared to compressive stress study. B. MDA-MB-231 (PIK3CAwt) cells were compressed using [7.0 to $8.0 \mathrm{kPa}$ ] high compressive stress(High) compared to no compression (None). GSEA KEGG NOTCH (47 genes), MAPK (264 genes) signaling pathways and REACTOME PI3K-AKT (102 genes) signaling in cancer were analyzed. p-values $<0.05$. NES: Normalized Enrichment Score; FDR: Fold Discovery Rate. C. MDA-MB-231 (PIK3CAwt; blue triangles) and MCF-7 (PIK3CAE545K mutated, black dots) breast cancer cells were gradually compressed ( $0 \mathrm{kPa}$; [0.3 to $0.8 \mathrm{kPa}$ ]; [1.5 to $4.0 \mathrm{kPa}$ ]; [7.0 to $8.0 \mathrm{kPa}]$ ) and gene expression was quantified using Agilent microarray (Data available in https://www.ncbi.nlm.nih.gov/geo/query/acc.cgi?acc=GSE133134). Differential expression analysis was performed and this data were correlated to GSEA KEGG NOTCH (47 genes), MAPK (264 genes) signaling pathways and REACTOME PI3K-AKT signaling in cancer (102 genes). \pm SEM. ${ }^{*}<0.05 ;{ }^{* *}<0.01 ;{ }^{* * *}<0.001$.

Figure 2. Comparative analysis between compressive stress, $\mathrm{PI} 3 \mathrm{~K} \alpha / \mathrm{PI} 3 \mathrm{~K} \beta$ signatures and REACTOME PI3K-AKT signaling in cancer. 1279, 933 and 1052 differentially expressed genes in PI3K $\alpha$ signature, PI3K $\beta$ signature, compressive stress and 102 genes of REACTOME PI3K-AKT signaling in cancer respectively, were compared to investigate overlapping. Venn diagram show the overlapping between PI3K $\alpha$ signature, PI3K $\beta$ signature, compressive stress and REACTOME PI3K-AKT signaling in cancer. Tables represent the overlapping between compressive stress/PI3K $\alpha$ signature (32 genes) (bottom left), compressive stress/PI3K $\beta$ signature (31 genes) (bottom right) and PI3K $\alpha / \mathrm{PI} 3 \mathrm{~K} \beta$ signatures (139 genes) (above center). The only common gene differentially expressed by PI3K $\alpha$ inhibition/PI3K $\beta$ inhibition/compressive stress was GABARAPL1 (highlighted in yellow).

Figure 3. Increased compressive stress influences PI3K and autophagy signaling pathways in breast cancer cells. MDA-MB-231 (PIK3CAwt; blue triangles) and MCF-7 (PIK3CAE545K mutated, black squares) breast cancer cells were gradually compressed ( $0 \mathrm{kPa}$; [0.3 to $0.8 \mathrm{kPa}$ ]; [1.5 to 4.0 $\mathrm{kPa}]$; [7.0 to $8.0 \mathrm{kPa}$ ] respectively) and gene expression was quantified using Agilent microarray (Data available in https://www.ncbi.nlm.nih.gov/geo/query/acc.cgi?acc=GSE133134). A. Differentially expressed genes ( $\mathrm{p}$-value $<0.05$ ) were correlated to $\mathrm{PI} 3 \mathrm{~K} \alpha / \beta$ signatures (PI3K $\alpha$ signature (1279 genes): after BYL719 inhibition; PI3K $\beta$ signature (933 genes): after AZD8186 inhibition). B. GABRAPL1 (1 gene) and autophagy (35 genes). \pm SEM. ${ }^{*}<0.05 ;{ }^{* * *}<0.001$. 
Di-Luoffo et al

\section{Discussion}

Results obtained in the current work reinforce the PI3K impact in mechanotransduction in cancer cells, the possible differential role of PI3K isoform specific signaling and the necessity to test the effect of PI3K $\alpha / \beta$ inhibitors in cancers submitted to compressive stress independently of their mutational status.

The PI3K pathway is essential for cell proliferation, survival, and metabolism [23, 24]. In human cancers, the PI3KCA gene is frequently mutated in particular in hot spot mutations that reside in the p $110 \alpha$ helical domain (E542K and E545K) found in over a third of estrogen receptor (ER)positive breast cancer, representing the most common genomic alteration in this group of tumors [25]. A large number of studies describe the effect of PI3K $\alpha / \beta$ inhibitions in different cancer models [10]. However, few high throughput sequencing data are available after PI3K inhibition and compressive stress. This limitation constrained our study to the analysis of different breast cancer cell lines in order to compare the combined effects of the PI3K $\alpha / \beta$ inhibitions and compressive stress. In our comparative study, two breast cancer cell lines were compared: MCF-7 containing a constitutive PI3 K $\alpha$ activation (PIK3CAE545K), and MDA-MB-231 with a wild-type PIK3CA and containing a high level of PTEN and low level of phosphorylated AKT [26]. PTEN phosphatase possesses a PI3K antagonist action balancing the activation of the canonical AKT-mTORC1 pathway [27]. Therefore, comparing MCF-7 and MDA-MB-231 allows to compare two cell lines with high and low activation of PI3K-AKT-mTORC1 axis respectively. We found that compressive stress affects PI3K-AKT pathway gene expression in PI3KCAwt cells, in contrast with constitutively active PI3K $\alpha$ cell line. Recent evidence demonstrates that PI3K enzymes are essential in mechanotransduction and we previously suggested that PI3K functions as a hub in mechanotransduction in pancreatic and breast cancers [8]. These data reinforce the importance of studying the coupling between mechanical stress and genetic alterations skewing pro-oncogenic cell signaling.

Increasing unidirectional compressive stress induced an overexpression of PI3K $\alpha$ and PI3K $\beta$ gene signatures. The PI3K isoform activation is notably cancer type- and context-dependent. In breast cancer cells, the increasing compression influence both the $\mathrm{PI} 3 \mathrm{~K} \alpha / \beta$ pathways gene expression, that are known to have different signaling outputs [28]. Our comparative analysis revealed that 1279 genes were significantly regulated after PI3K $\alpha$ inhibition, 933 after PI3K $\beta$ inhibition and 1052 in compressive stress condition, therefore 139 commonly regulated when inhibiting PI3K $\alpha$ and PI3K $\beta$. These data reinforce the importance of studying the isoform specificity of PI3K in a compressive stress environment.

Finally, by comparing PI3K $\alpha$ and PI3K $\beta$ inhibitions, compressive stress- regulated genes and reactome PI3K-AKT signaling in cancer pathway lists of genes, only GABARAPL1 gene was significantly affected and overlap in these four studies. GABARAPL1 encodes for a protein cleaved by ATG4B protease before its conjugation to phospholipids. This modified, lipidated form is localized on the surface of autophagosomes and lysosomes, participating in their formation and promote tubulin polymerization and bundling to form microtubules and therefore involved in autophagy process (reviewed in [22]). Autophagy is a highly conserved 
Di-Luoffo et al

catabolic process, participating in the balanced cell proliferation/death in tumor and the tumor microenvironment regulation. This process is highly regulated by PI3K-AKT-mTOR pathway in various tumors. We know that inhibition of PI3K-AKT-mTOR induces autophagy as part of a tumor growth suppressor in early cancerogenesis [29,30]. This pathway is known as a key pathway in therapeutic strategy for a variety of tumors. Further, we know that induction of autophagy is correlated with mechanical stress [20,31]. In Dictyostelium discoideum, an organism which do not present any homolog of vertebrate Class II PI3K, compression activates autophagy in a mTORC1 independent manner [32]. This mechanism, if confirmed in mammalian cells, could participate in cancer cell survival as well as resistance to chemical treatments.

Under increasing compression, gene expression signatures of different protumoral signaling pathways were significantly enriched. However and surprisingly, YAP/TAZ via Hippo or No Hippo pathway, known as key regulator of mechanotransduction under tensile stress [33], was not significantly affected in both PIK3CAE545K and PIK3CAWT breast cancer cells under unidirectional compressive stress (Figure. S1B). In compressive stress context, the implication of YAP/TAZ pathway was less investigated. It was also assumed that this stress would induce the same signal pathways than tensile stress. This assumption could have been prompted by the fact that some techniques used to induce compression could also promote tensile stress [34, 35]. In Kim et al. 2019, cells were compressed in vitro using alginate disk and compression cylinder. The tension context, due to cell-cell or cell-coating adhesions, is almost absent in these experimental conditions [16]. However, a stress associated with cytoskeleton remodeling cannot be completely ruled out in these conditions. The exact type of mechanical stress and the celldependent induction of cytoskeleton is key to understand cell mechanotransduction processes, as cells adapt to them environmental stresses. Further, class I PI3Ks were described as upstream activators of the YAP/TAZ pathway; tensile stress is permissive for their control of YAP/TAZ and targeting of PI3K could be a novel strategy to hinder the potential YAP/TAZ oncogenic dependence [8]. This PI3K activation was described in tensile stress conditions, however our transcriptional analysis does not seem to confirm those finding in the context of unidirectional compressive stress.

In breast cancer, compressive stress is likely to occur as it was associated with large fibrosis and extracellular matrix (ECM) deposits [36]. Similarly, in pancreatic cancer, there is an important influence of tumoral microenvironment possessing a variable stiffness and variable interstitial pressure depending on the area in pancreas and progress statement of disease [37]. In addition to compressive stress induced by ECM, ECM also influences the desmoplastic reaction via epithelial-mesenchymal transition (EMT) and further resistance to chemotherapeutic agents [38]. Because those high compressive stress occurs in breast cancer [39] but also in pancreatic ductal adenocarcinomas [12], our study should be extended to other types of solid cancers.

\section{Conclusion}

In addition to the canonical pathways (YAP/TAZ and Piezo) associated with mechanical stresses, our study provides transcriptomic evidences for the role of PI3K-AKT pathway in compressioninduced mechanotransduction, through class I PI3Ks (PI3K $\alpha$-PI3K $\beta$ ) roles. Further, other mechanisms inherent to compressive stress, such as autophagy induction, may provide a proliferative advantage and increase cell resistance in compression stress environment. Finally, oncogenic alterations are known to skew normal cell signaling. However, environmental context 
Di-Luoffo et al

such as compressive stress could also activate oncogenic pathways and induce novel tumoral vulnerabilities independently from their mutational status.

\section{Materials and Methods}

Differential expression analysis after compressive stress in MCF-7 and MDA-MB-231 were performed from data provided by Kim et al., 2019 [16]. MCF-7 and MDA-MB-231 breast cancer cells were gradually compressed $(0 ; 0.3$ to $0.8 ; 1.5$ to $4.0 ; 7.0$ to $8.0 \mathrm{kPa}$ ) and gene expression was quantified using Agilent-039494 SurePrint G3 Human GE v2 8x60K Microarray 039381, data available at https://www.ncbi.nlm.nih.gov/geo/query/acc.cgi?acc=GSE133134. The two $\mathrm{PI} 3 \mathrm{~K} \alpha$ and PI3K $\beta$ inhibition signatures were performed from differential expression analysis after PI3K $\alpha$ inhibition with BYL-719 (1 $\mu \mathrm{M}$ for 16 to 48 hours) in MCF-7 breast cancer cells (https://www.ncbi.nlm.nih.gov/geo/query/acc.cgi?acc=GSE64033) [17] or PI3K $\beta$ inhibition after AZD8186 treatment $(100 \mathrm{mg} / \mathrm{kg}$ twice daily for 5 days $)$ in HCC70 cells (https://www.ebi.ac.uk/arrayexpress/experiments/E-MTAB-4656/) [18]. For compressive stress and $\mathrm{PI} 3 \mathrm{~K} \alpha$ and $\mathrm{PI} 3 \mathrm{~K} \beta$ inhibition signatures, differential expression analysis were performed using DESeq2 v1.26.0 [40] on $\mathrm{R}$ version 3.6 .3 with adjusted $\mathrm{p}$-value $<0.05$. All differential expression data are relative data from normalized transcript per million (TPM). Pvalues on graphs were calculated using t-test comparing no compression condition $(0 \mathrm{kPa})$ to each compression condition ([0.3-0.8], [1.5-4.0] or [7.0-8.0] kPa) ${ }^{*}<0.05 ;{ }^{* *}<0.01 ;{ }^{* * *}<0.001$. Enrichement analysis were performed using Gene Set Enrichement Analysis software (GSEA), pvalue $<0.05$ and Fold Discovery Rate (FDR) $<25 \%$.

\section{Supplementary Materials:}

Figure S1. Housekeeping genes and YAP/TAZ pathway under increasing compressive stress in breast cancer cells. MDA-MB-231 (PIK3CAwt; blue triangles) and MCF-7 (PIK3CAE545K mutated, black squares) breast cancer cells were gradually compressed (0 kPa; [0.3 to $0.8 \mathrm{kPa}$; [ 1.5 to 4.0 $\mathrm{kPa}$; [ 7.0 to $8.0 \mathrm{kPa}$ ] respectively) and gene expression was quantified using Agilent microarray (Data available in https://www.ncbi.nlm.nih.gov/geo/query/acc.cgi?acc=GSE133134). Differentially expressed genes ( $p$-value $<0.05$ ) were correlated. A. ACTINB (1 gene) and LAMINA1 (1 gene) and LAMIN gene class (12 genes) as housekeeping genes. B. Mechanoregulation and pathology of YAP/TAZ via HIPPO and non HIPPO mechanisms (45 genes). \pm SEM. ${ }^{*}<0.05 ;{ }^{* *}<0.01 ;{ }^{* * *}<0.001$.

Author Contributions: Analysis and interpretation of data: MDL and JGG. Drafting of the manuscript: MDL and JGG. Revising the manuscript: MDL, MD and JGG. Obtained funding: JGG and MD.

Funding: Our work on this topic is funded by Fondation Toulouse Cancer Santé (Mecharesist) and Inserm Plan Cancer (PressDiagTherapy).

Data Availability Statement:

For this work, we utilized transcriptomic data from compressed breast cancer cells from Kim et al., 2019 [16], from Gene Expression Omnibus GEO accession number: GSE133134. Our work 
Di-Luoffo et al

cor-relates compression data to PI3K $\alpha$ inhibition data from Bosch et al., 2015 [17], GEO accession number: GSE64033) or PI3K $\beta$ inhibition data from Lynch et al., 2017 [18], ArrayExpress: ENA - ERP015852).

Acknowledgments: We thank our colleagues for their critical reading of the manuscript. Conflicts of Interest: The authors disclose no conflicts.

\section{References}

1. Northcott, J. M.; Dean, I. S.; Mouw, J. K.; Weaver, V. M., Feeling Stress: The Mechanics of Cancer Progression and Aggression. Front Cell Dev Biol 2018, 6, 17.

2. $\quad$ Levental, K. R.; Yu, H.; Kass, L.; Lakins, J. N.; Egeblad, M.; Erler, J. T.; Fong, S. F.; Csiszar, K.; Giaccia, A.; Weninger, W.; Yamauchi, M.; Gasser, D. L.; Weaver, V. M., Matrix crosslinking forces tumor progression by enhancing integrin signaling. Cell 2009, 139, (5), 891-906.

3. Delarue, M.; Montel, F.; Vignjevic, D.; Prost, J.; Joanny, J. F.; Cappello, G., Compressive stress inhibits proliferation in tumor spheroids through a volume limitation. Biophys J 2014, 107, (8), 1821-1828.

4. Kalli, M.; Minia, A.; Pliaka, V.; Fotis, C.; Alexopoulos, L. G.; Stylianopoulos, T., Solid stressinduced migration is mediated by GDF15 through Akt pathway activation in pancreatic cancer cells. Sci Rep 2019, 9, (1), 978.

5. Rizzuti, I. F.; Mascheroni, P.; Arcucci, S.; Ben-Meriem, Z.; Prunet, A.; Barentin, C.; Riviere, C.; Delanoe-Ayari, H.; Hatzikirou, H.; Guillermet-Guibert, J.; Delarue, M., Mechanical Control of Cell Proliferation Increases Resistance to Chemotherapeutic Agents. Phys Rev Lett 2020, 125, (12), 128103.

6. $\quad$ Cobbaut, M.; Karagil, S.; Bruno, L.; Diaz de la Loza, M. D. C.; Mackenzie, F. E.; Stolinski, M.; Elbediwy, A., Dysfunctional Mechanotransduction through the YAP/TAZ/Hippo Pathway as a Feature of Chronic Disease. Cells 2020, 9, (1).

7. Piccolo, S.; Dupont, S.; Cordenonsi, M., The biology of YAP/TAZ: hippo signaling and beyond. Physiol Rev 2014, 94, (4), 1287-312.

8. Di-Luoffo, M.; Ben-Meriem, Z.; Lefebvre, P.; Delarue, M.; Guillermet-Guibert, J., PI3K functions as a hub in mechanotransduction. Trends Biochem Sci 2021.

9. Vanhaesebroeck, B.; Guillermet-Guibert, J.; Graupera, M.; Bilanges, B., The emerging mechanisms of isoform-specific PI3K signalling. Nat Rev Mol Cell Biol 2010, 11, (5), 329-41.

10. Arcucci, S.; Ramos-Delgado, F.; Cayron, C.; Therville, N.; Gratacap, M. P.; Basset, C.; Thibault, B.; Guillermet-Guibert, J., Organismal roles for the PI3Kalpha and beta isoforms: their specificity, redundancy or cooperation is context-dependent. Biochem J 2021, 478, (6), 11991225.

11. Pons-Tostivint, E.; Thibault, B.; Guillermet-Guibert, J., Targeting PI3K Signaling in Combination Cancer Therapy. Trends Cancer 2017, 3, (6), 454-469.

12. Thibault, B.; Ramos-Delgado, F.; Pons-Tostivint, E.; Therville, N.; Cintas, C.; Arcucci, S.; Cassant-Sourdy, S.; Reyes-Castellanos, G.; Tosolini, M.; Villard, A. V.; Cayron, C.; Baer, R.; 
Di-Luoffo et al

Bertrand-Michel, J.; Pagan, D.; Ferreira Da Mota, D.; Yan, H.; Falcomata, C.; Muscari, F.; Bournet, B.; Delord, J. P.; Aksoy, E.; Carrier, A.; Cordelier, P.; Saur, D.; Basset, C.; Guillermet-Guibert, J., Pancreatic cancer intrinsic PI3Kalpha activity accelerates metastasis and rewires macrophage component. EMBO Mol Med 2021, 13, (7), e13502.

13. Zhao, Y.; Montminy, T.; Azad, T.; Lightbody, E.; Hao, Y.; SenGupta, S.; Asselin, E.; Nicol, C.; Yang, X., PI3K Positively Regulates YAP and TAZ in Mammary Tumorigenesis Through Multiple Signaling Pathways. Mol Cancer Res 2018, 16, (6), 1046-1058.

14. Ellis, H.; Ma, C. X., PI3K Inhibitors in Breast Cancer Therapy. Curr Oncol Rep 2019, 21, (12), 110.

15. Stylianopoulos, T., The Solid Mechanics of Cancer and Strategies for Improved Therapy. J Biomech Eng 2017, 139, (2).

16. Kim, B. G.; Sung, J. S.; Jang, Y.; Cha, Y. J.; Kang, S.; Han, H. H.; Lee, J. H.; Cho, N. H., Compression-induced expression of glycolysis genes in CAFs correlates with EMT and angiogenesis gene expression in breast cancer. Commun Biol 2019, 2, 313.

17. Bosch, A.; Li, Z.; Bergamaschi, A.; Ellis, H.; Toska, E.; Prat, A.; Tao, J. J.; Spratt, D. E.; ViolaVillegas, N. T.; Castel, P.; Minuesa, G.; Morse, N.; Rodon, J.; Ibrahim, Y.; Cortes, J.; Perez-Garcia, J.; Galvan, P.; Grueso, J.; Guzman, M.; Katzenellenbogen, J. A.; Kharas, M.; Lewis, J. S.; Dickler, M.; Serra, V.; Rosen, N.; Chandarlapaty, S.; Scaltriti, M.; Baselga, J., PI3K inhibition results in enhanced estrogen receptor function and dependence in hormone receptor-positive breast cancer. Sci Transl Med 2015, 7, (283), 283ra51.

18. Lynch, J. T.; Polanska, U. M.; Delpuech, O.; Hancox, U.; Trinidad, A. G.; Michopoulos, F.; Lenaghan, C.; McEwen, R.; Bradford, J.; Polanski, R.; Ellston, R.; Avivar-Valderas, A.; Pilling, J.; Staniszewska, A.; Cumberbatch, M.; Critchlow, S. E.; Cruzalegui, F.; Barry, S. T., Inhibiting PI3Kbeta with AZD8186 Regulates Key Metabolic Pathways in PTEN-Null Tumors. Clin Cancer Res 2017, 23, (24), 7584-7595.

19. Cairns, R. A.; Harris, I. S.; Mak, T. W., Regulation of cancer cell metabolism. Nat Rev Cancer 2011, 11, (2), 85-95.

20. Xu, Z.; Han, X.; Ou, D.; Liu, T.; Li, Z.; Jiang, G.; Liu, J.; Zhang, J., Targeting PI3K/AKT/mTORmediated autophagy for tumor therapy. Appl Microbiol Biotechnol 2020, 104, (2), 575-587.

21. Heras-Sandoval, D.; Perez-Rojas, J. M.; Hernandez-Damian, J.; Pedraza-Chaverri, J., The role of $\mathrm{PI} 3 \mathrm{~K} / \mathrm{AKT} / \mathrm{mTOR}$ pathway in the modulation of autophagy and the clearance of protein aggregates in neurodegeneration. Cell Signal 2014, 26, (12), 2694-701.

22. Le Grand, J. N.; Chakrama, F. Z.; Seguin-Py, S.; Fraichard, A.; Delage-Mourroux, R.; Jouvenot, M.; Boyer-Guittaut, M., GABARAPL1 (GEC1): original or copycat? Autophagy 2011, 7, (10), 1098-107.

23. Engelman, J. A.; Luo, J.; Cantley, L. C., The evolution of phosphatidylinositol 3-kinases as regulators of growth and metabolism. Nat Rev Genet 2006, 7, (8), 606-19.

24. Cantley, L. C., The phosphoinositide 3-kinase pathway. Science 2002, 296, (5573), 16557.

25. Samuels, Y.; Wang, Z.; Bardelli, A.; Silliman, N.; Ptak, J.; Szabo, S.; Yan, H.; Gazdar, A.; Powell, S. M.; Riggins, G. J.; Willson, J. K.; Markowitz, S.; Kinzler, K. W.; Vogelstein, B.; Velculescu, 
Di-Luoffo et al

V. E., High frequency of mutations of the PIK3CA gene in human cancers. Science 2004, 304, (5670), 554.

26. Weng, L. P.; Smith, W. M.; Dahia, P. L.; Ziebold, U.; Gil, E.; Lees, J. A.; Eng, C., PTEN suppresses breast cancer cell growth by phosphatase activity-dependent G1 arrest followed by cell death. Cancer Res 1999, 59, (22), 5808-14.

27. Carracedo, A.; Pandolfi, P. P., The PTEN-PI3K pathway: of feedbacks and cross-talks. Oncogene 2008, 27, (41), 5527-41.

28. Cintas, C.; Douche, T.; Dantes, Z.; Mouton-Barbosa, E.; Bousquet, M. P.; Cayron, C.; Therville, N.; Pont, F.; Ramos-Delgado, F.; Guyon, C.; Garmy-Susini, B. H.; Cappello, P.; BurletSchiltz, O.; Hirsch, E.; Gomez-Brouchet, A.; Thibault, B.; Reichert, M.; Guillermet-Guibert, J., Phosphoproteomics identifies PI3K inhibitor-selective adaptive responses in pancreatic cancer cell therapy and resistance. Mol Cancer Ther 2021.

29. Cui, L. H.; Li, C. X.; Zhuo, Y. Z.; Yang, L.; Cui, N. Q.; Zhang, S. K., Saikosaponin d ameliorates pancreatic fibrosis by inhibiting autophagy of pancreatic stellate cells via PI3K/Akt/mTOR pathway. Chem Biol Interact 2019, 300, 18-26.

30. Yang, J.; Pi, C.; Wang, G., Inhibition of PI3K/Akt/mTOR pathway by apigenin induces apoptosis and autophagy in hepatocellular carcinoma cells. Biomed Pharmacother 2018, 103, 699-707.

31. Claude-Taupin, A.; Codogno, P.; Dupont, N., Links between autophagy and tissue mechanics. J Cell Sci 2021, 134, (17).

32. King, J. S.; Veltman, D. M.; Insall, R. H., The induction of autophagy by mechanical stress. Autophagy 2011, 7, (12), 1490-9.

33. Totaro, A.; Panciera, T.; Piccolo, S., YAP/TAZ upstream signals and downstream responses. Nat Cell Biol 2018, 20, (8), 888-899.

34. Kalli, M.; Voutouri, C.; Minia, A.; Pliaka, V.; Fotis, C.; Alexopoulos, L. G.; Stylianopoulos, T., Mechanical Compression Regulates Brain Cancer Cell Migration Through MEK1/Erk1 Pathway Activation and GDF15 Expression. Front Oncol 2019, 9, 992.

35. Chen, Y.; Li, Z.; Ju, L. A., Tensile and compressive force regulation on cell mechanosensing. Biophys Rev 2019, 11, (3), 311-318.

36. Piersma, B.; Hayward, M. K.; Weaver, V. M., Fibrosis and cancer: A strained relationship. Biochim Biophys Acta Rev Cancer 2020, 1873, (2), 188356.

37. Provenzano, P. P.; Cuevas, C.; Chang, A. E.; Goel, V. K.; Von Hoff, D. D.; Hingorani, S. R., Enzymatic targeting of the stroma ablates physical barriers to treatment of pancreatic ductal adenocarcinoma. Cancer Cell 2012, 21, (3), 418-29.

38. Rice, A. J.; Cortes, E.; Lachowski, D.; Cheung, B. C. H.; Karim, S. A.; Morton, J. P.; Del Rio Hernandez, A., Matrix stiffness induces epithelial-mesenchymal transition and promotes chemoresistance in pancreatic cancer cells. Oncogenesis 2017, 6, (7), e352.

39. Verret, B.; Cortes, J.; Bachelot, T.; Andre, F.; Arnedos, M., Efficacy of PI3K inhibitors in advanced breast cancer. Ann Oncol 2019, 30, (Suppl_10), x12-x20.

40. Anders, S.; Huber, W., Differential expression analysis for sequence count data. Genome Biol 2010, 11, (10), R106. 
bioRxiv preprint doi: https://doi.org/10.1101/2021.10.18.464825; this version posted October 19, 2021. The copyright holder for this preprint (which was not certified by peer review) is the author/funder. All rights reserved. No reuse allowed without permission.

Di-Luoffo et al 


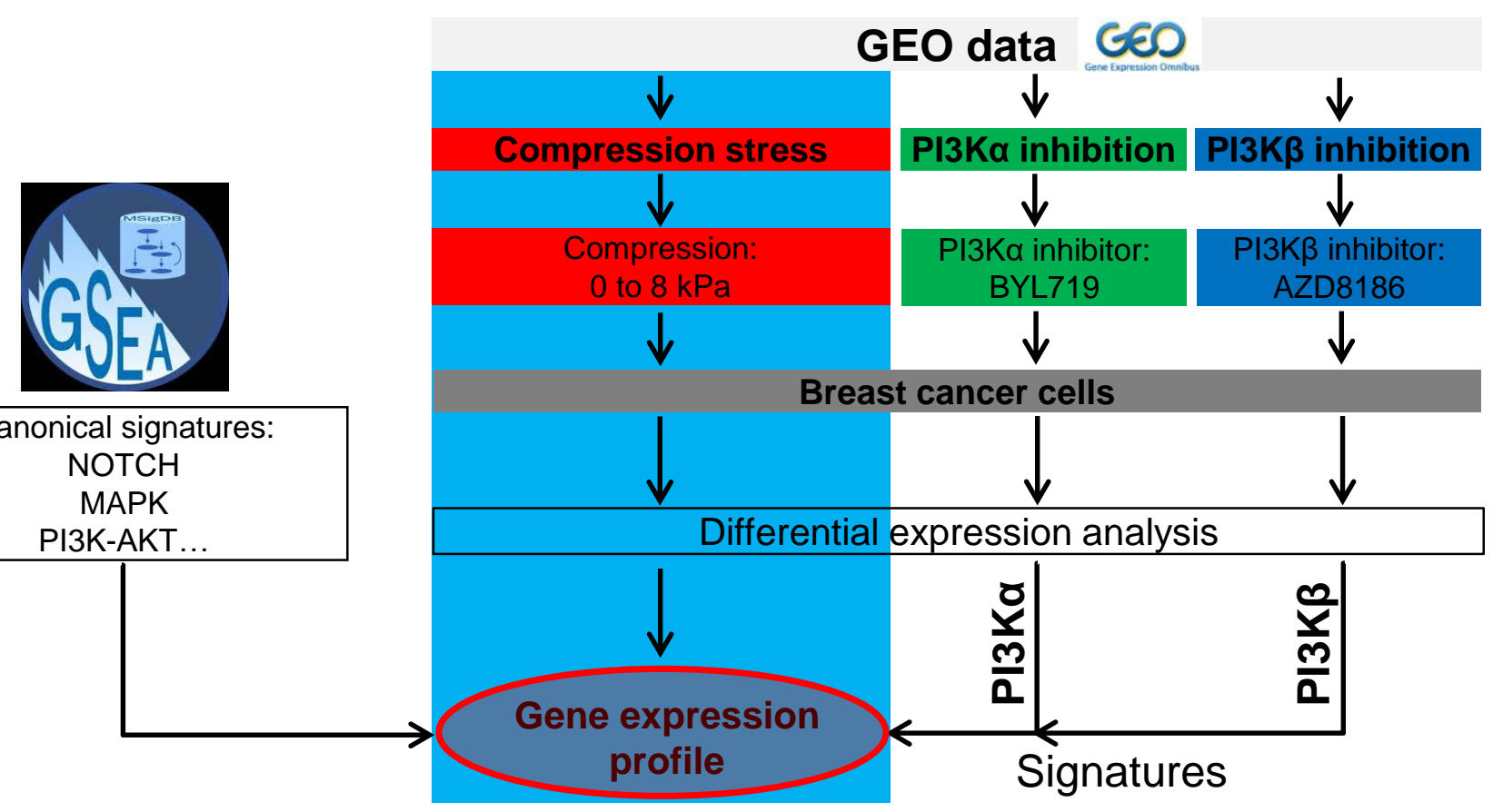

\section{Canonical method}

B
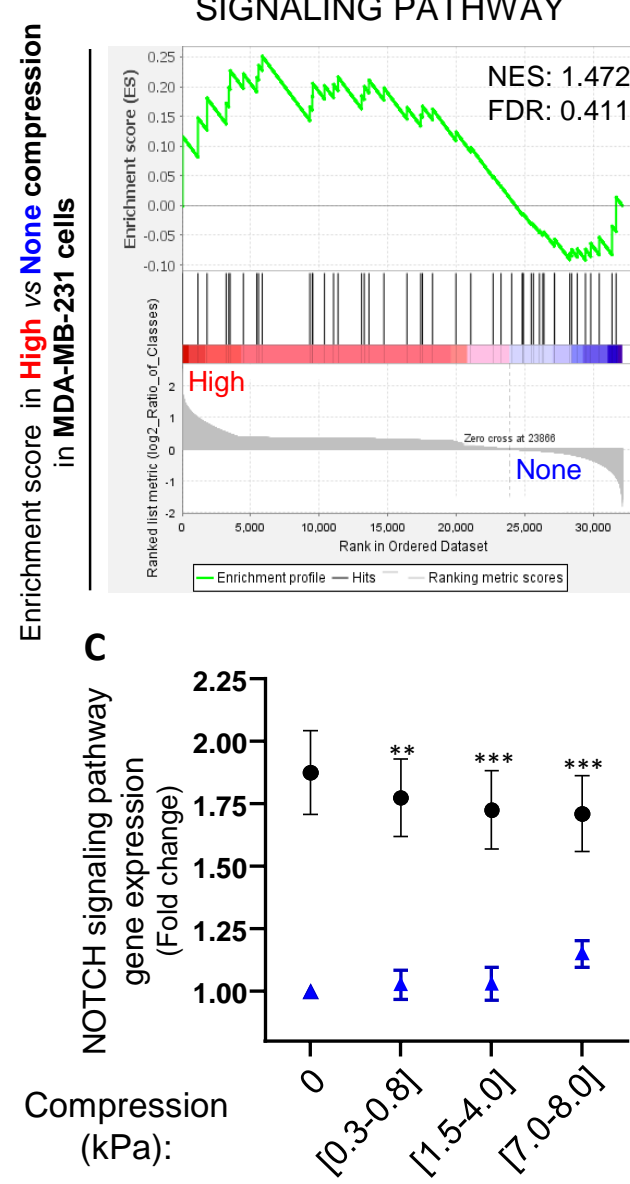

KEGG NOTCH

SIGNALING PATHWAY

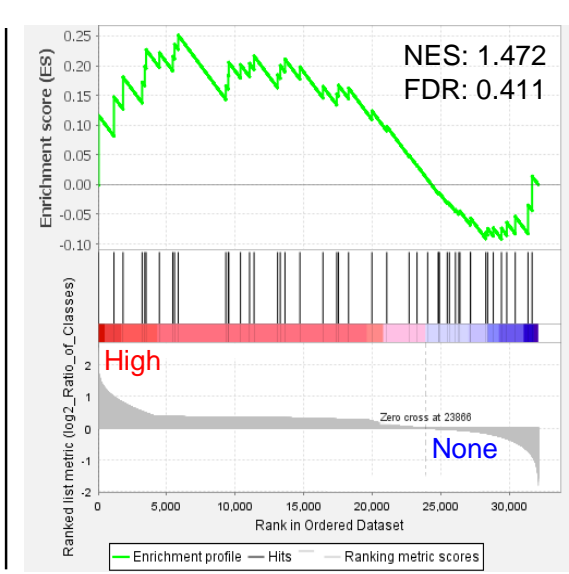

KEGG MAPK

SIGNALING PATHWAY
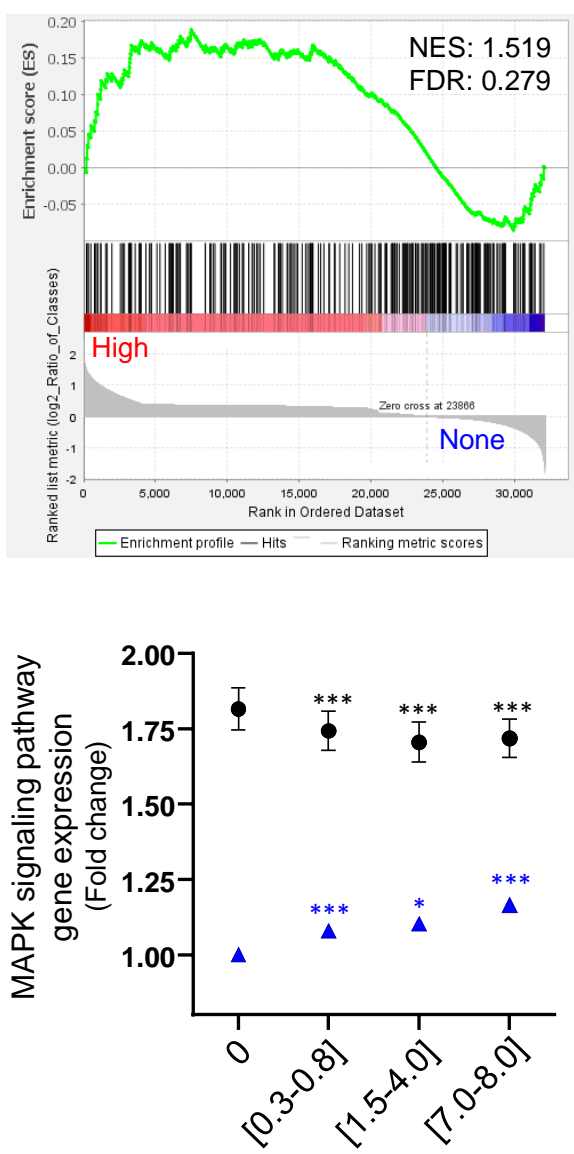

\section{Our analysis}

REACTOME PI3K-AKT SIGNALING IN CANCER

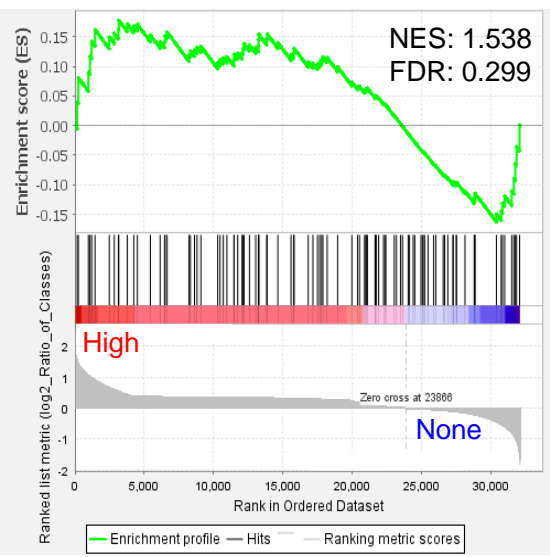




\begin{tabular}{|c|c|c|c|c|c|c|c|}
\hline \multicolumn{7}{|c|}{ PI3Ka/PI3K $\beta$} \\
\hline GABARAPL1 & C16orf61 & CENPB & EIF3B & GRWD1 & MRPL14 & NUP54 & PSMD12 \\
\hline ABCA1 & C16orf8 & CIRH1A & ELF3 & GTPBP4 & MRPL3 & NXT1 & PSMG1 \\
\hline ABCE1 & C16orf87 & COL12A1 & FABP5 & HBP1 & MRPL36 & ODC1 & PSMG3 \\
\hline ABCF2 & C10r135 & CORO1C & FAM134B & HIGD1A & MRTO4 & OTUD1 & PTS \\
\hline ACAT2 & C20orf24 & CRBN & FAM49B & HMGCS1 & MVD & PAK1IP1 & RANBP1 \\
\hline ACLY & C3orf14 & CSE1L & FBXO32 & HSD17B12 & MYLIP & PCMTD1 & RAP2C \\
\hline ADSL & C5orf41 & CTDSP2 & FDFT1 & HSPA4 & NDUFC1 & PCMTD2 & RB1CC1 \\
\hline AKR1C3 & C7orf41 & CTPS & FDPS & IDI1 & NIP7 & PDK4 & RBL2 \\
\hline ALDH1B1 & CA2 & DDIT4 & FH & IMP4 & NIPA2 & PDSS1 & RDH11 \\
\hline AMD1 & CALCOC01 & DEGS1 & FOXO1 & INSIG1 & NME1 & PHF23 & RHOC \\
\hline ATAD3A & CCNE1 & DHCR24 & FOXO4 & IRS2 & NOP2 & PHLDA2 & RNASEH1 \\
\hline BCAS1 & CCNG2 & DHCR7 & GABPB1 & JMY & NOP56 & PIK3IP1 & RPF2 \\
\hline BOLA3 & CCRN4L & DKC1 & GBP2 & KIAA0430 & NOP58 & PIM1 & RPL39L \\
\hline BRD8 & CCT2 & DSCC1 & GCH1 & KLHL24 & NPHP3 & PNO1 & \\
\hline BRI3BP & CCT7 & DUSP14 & GGCT & LDLR & NSDHL & PPAT & \\
\hline BRIX1 & CDC42EP2 & DYNLL1 & GLRX2 & LRP8 & NSUN2 & PPIL1 & \\
\hline BYSL & CDK5RAP3 & EBP & GRB2 & MAGEF1 & NUMA1 & PPP2CA & \\
\hline C13orf15 & CENPB & EIF2B3 & GRB7 & MCM10 & NUP35 & PSMC2 & \\
\hline
\end{tabular}

\begin{tabular}{|c|}
\hline Comp stress/PI3Ka \\
\hline GABARAPL1 \\
\hline AK2 \\
\hline CACYBP \\
\hline CDC25A \\
\hline CDH26 \\
\hline CHORDC1 \\
\hline CXCR7 \\
\hline CYP26B1 \\
\hline DOLK \\
\hline EIF5 \\
\hline FLJ10661 \\
\hline GATS \\
\hline KREMEN2 \\
\hline LCMT2 \\
\hline LFNG \\
\hline MRPL1 \\
\hline PARD6G \\
\hline PDE12 \\
\hline POLR3H \\
\hline RBM14 \\
\hline RIN2 \\
\hline RNU1-5 \\
\hline RPUSD2 \\
\hline RTN4IP1 \\
\hline SCARNA13 \\
\hline SEMA6C \\
\hline SESTD1 \\
\hline SNORA12 \\
\hline SPATA7 \\
\hline SPRED1 \\
\hline TTC21A \\
\hline \\
\hline
\end{tabular}

PI3Ka (1279 genes)

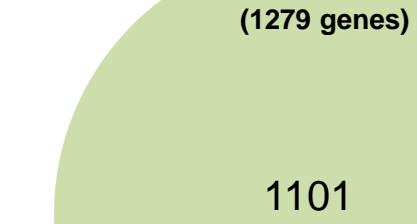

139
PI3K-AKT
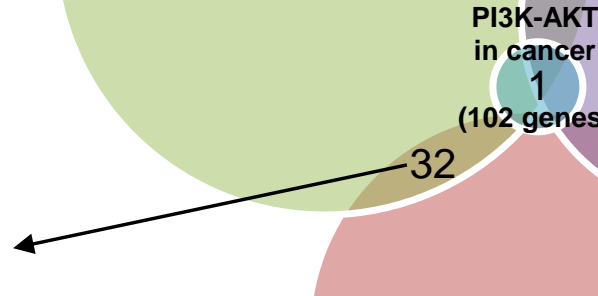

102 genes)

PI3K $\beta$

(933 genes)

754

31
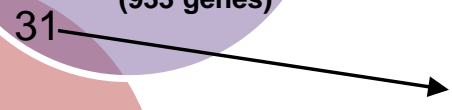

$\rightarrow$

973

Compressive Stress

(1052 genes)

\begin{tabular}{|c|}
\hline Comp stress/PI3K $\beta$ \\
\hline GABARAPL1 \\
\hline ANKRD36 \\
\hline C7orf74 \\
\hline CLK4 \\
\hline CTGF \\
\hline DDX58 \\
\hline ERRF11 \\
\hline F3 \\
\hline FAM13A \\
\hline FAS \\
\hline GDA \\
\hline INPP4B \\
\hline LOC100272228 \\
\hline LOC100289230 \\
\hline LSS \\
\hline MAT2A \\
\hline P2RX7 \\
\hline PNPLA3 \\
\hline POP1 \\
\hline S100PBP \\
\hline SESTD1 \\
\hline SNORD86 \\
\hline SPATA7 \\
\hline SYTL4 \\
\hline TIA1 \\
\hline TRAF5 \\
\hline TRANK1 \\
\hline ZNF248 \\
\hline ZNF302 \\
\hline ZNF37BP \\
\hline \\
\hline
\end{tabular}


PI3Ka signature

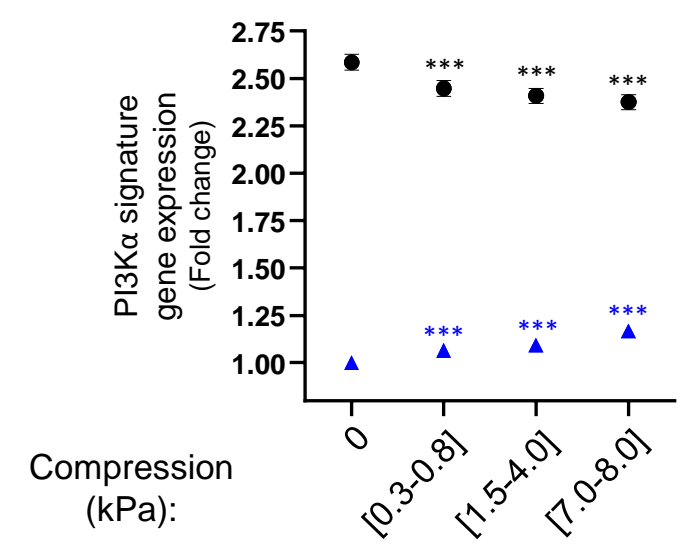

B

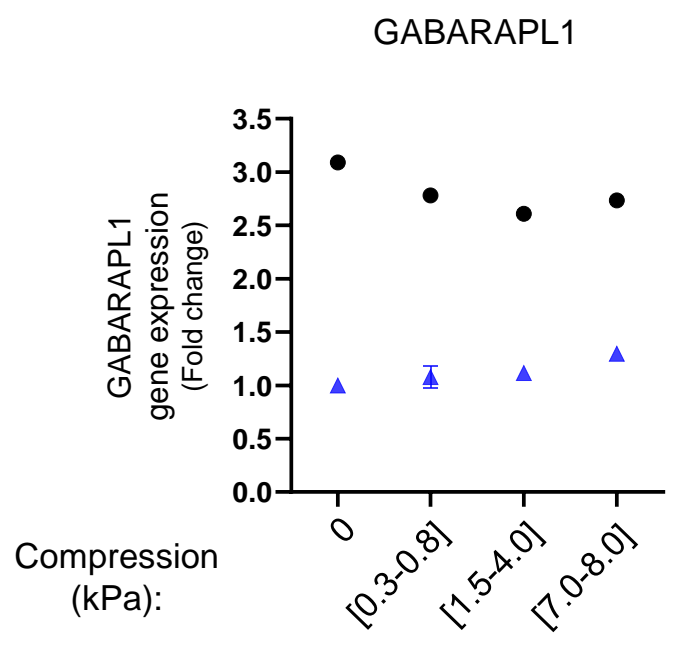

PI3K $\beta$ signature

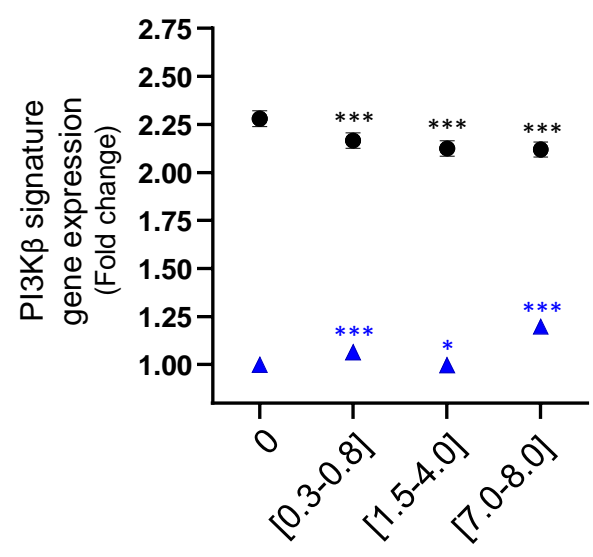

AUTOPHAGY

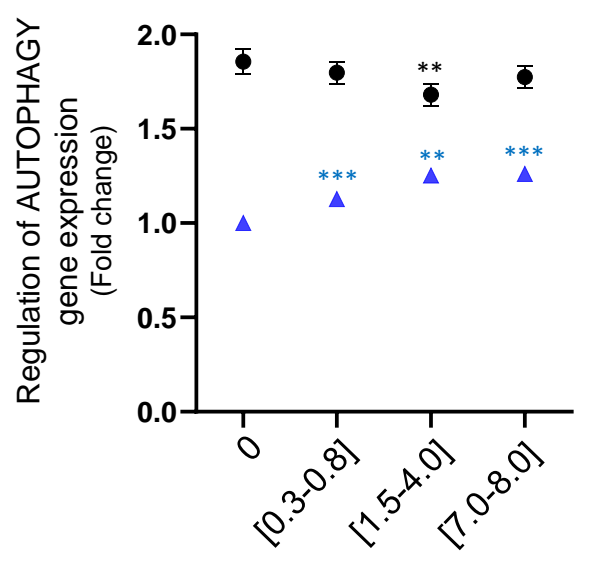

- MCF-7 (PIK3CAE545K mut)

- MDA-MB-231 (PIK3CA wt) 